\title{
The Potential Tumor Promotional Role of circVAPA in Retinoblastoma via Regulating miR-615-3p and SMARCEI
}

\section{Qibin Xu (D)}

Department of Ophthalmology, Zhejiang Hospital of Integrated Traditional Chinese and Western Medicine (Hangzhou Red Cross Hospital), Hangzhou, Zhejiang Province, People's Republic of China
This article was published in the following Dove Press journal: OncoTargets and Therapy

Background: Growing evidence reveals that circ development. However, the effects and possible echani is of circR.As in retinoblastoma (RB) are far from clear.

Methods: circVAPA expression patte vas identif by A-qPCR. circVAPA induced effects on RB cells were tested by CK- clone formı, flow cytometry and transwell assays. Bioinformatics assay, rescue experime and dual-luciferase tests were applied for mechanism exploration. Add onalry, mouse mod were established for in vivo assays.

Results: circVAPA was upr alated in hum $\mathrm{RB}$ specimen and RB cell lines, and was correlated with poor outcomes of $\mathrm{Rb}$ atients. Knod lown of circVAPA could suppress the malignant phenotypes of RB. Mechanis experim is demonstrated that miR-615-3p could reverse the circVAPA induce on RB cerro, and the downstream oncogene SMARCE1 was positively regulated by circVk A via $5-3 p$. Further, in vivo analysis confirmed the findings.

Concly In sur circVAPA promoted RB proliferation and metastasis by sponging miR 15-3p, hereby regulating SMARCE1. CircVAPA was a potential biomarker for $\mathrm{Rb}$ apy.

Ke , s: circular RNA, retinoblastoma, $\operatorname{circVAPA}, \operatorname{miR}-615-3 p, S M A R C E 1$

\section{troduction}

Ret. oblastoma (RB) is the most common malignant tumor in children under five years old. ${ }^{1} \mathrm{RB}$ is not sensitive to radiotherapy and chemotherapy. Although great efforts have been made to tackle this disease, the survival rate is under $5 \%$ in developing countries. ${ }^{2}$ Hence, exploring biological mechanisms of RB progression and finding out biomarkers and therapeutic approach are urgent for diagnose and therapy of this disease.

Circular RNAs (circRNAs) are a subclass of non-coding RNAs with single-strands, and attracted great attention recently. ${ }^{3,4}$ Most circRNAs are generated from exons with no protein-coding ability. ${ }^{5,6}$ CircRNAs were reported predominantly located in cytoplasm, where they sponge miRNAs, thereby releasing the target genes sequestered by miRNAs. ${ }^{7,8}$ Emerging evidence has reported the important roles of circRNAs in diverse cancer types. ${ }^{9}$ However, the function of circRNAs in RB has been rarely reported. circVAPA, derived from vesicle-associated membrane protein-associated protein A, was a novel circular RNA that was recently found associated with cancer progression. $\operatorname{circ} V A P A$ was first found to play roles in colorectal cancer via interacting with $m i R-101 .{ }^{10}$ Liu et al observed that $\operatorname{circVAPA}$ was upregulated in hepatocellular cancer and enhanced cell proliferation. ${ }^{11}$ Zhou et al observed that circVAPA played as a
Department of Ophthalmology, Zhejiang Hospital of Integrated Traditional Chinese and Western Medicine (Hangzhou Red Cross Hospital), 208 Huancheng Road East, Hangzhou, Zhejiang Province 310003, People's Republic of China Tel +8613989872710

Email qibinxueyes@163.com 
tumor promoter via modulating $m i R-1615-5 p$ in breast cancer development. ${ }^{12}$ Nevertheless, the role of circVAPA in other cancer types has not been reported, including in RB.

In our work, we observed the overexpression of $\operatorname{circ} V A P A$ in RB tissues, as well as the oncogenic effects on RB cells by promoting cell proliferation, migration and invasion. Further, the underlying molecular mechanism was explored and we revealed that circVAPA acted as an oncogene in RB progression via sponging $m i R-615-3 p$, thereby positively modulating SMARCE1. This research may help provide novel targets for RB clinical diagnosis and therapy.

\section{Methods}

\section{Clinical Samples}

We obtained 50-paired RB tissue samples and adjacent normal ones from Zhejiang Hospital of Integrated Traditional Chinese and Western Medicine. The involved patients received surgery between 2017 and 2019. We obtained written informed consent from every participant, and this study was approved by the Ethics Committee of Zhejiang Hospital of Integrated Traditional Chinese and Western Medicine. Our study was conducted in accordance with the Declaration of Helsinki.

\section{Cell Culture}

Human retinal pigment epithelial cell line $(\mathrm{AB}$ and human RB cell lines (WERI Rb1, hTERT-RP A, SO- B-50 and Y79) were provided by Cell Bank

Chinese Academy of Sciences (Shang' A, China, ells were subjected to RPMI-1640 medium a o, USA) b $10 \%$ FBS (Gibco, USA). Incubation was main, ned at $37^{\circ} \mathrm{C}$ with $5 \% \mathrm{CO}_{2}$.

Table I Primers of qRT-P

\begin{tabular}{|c|c|c|}
\hline Gene & & \\
\hline circVAPA & & $\begin{array}{l}\text { 5'-GTGTCTGGCAAGGAACACTA-3' } \\
\text { 5'-GGTGGAGAAGAGGGACAATAAG-3' }\end{array}$ \\
\hline$m i R-6 / 5-3 p$ & $\begin{array}{l}\text { tem-loop RT primer } \\
\text { Purward } \\
\text { Reverse }\end{array}$ & $\begin{array}{l}\text { 5'- GTCGTATCCAGTGCAGGGTCCGAGGTATTCGCACTGGATACGACAGGCUC -3' } \\
\text { 5' UUCUCCCUCUGGGUCC-3' } \\
\text { 5'-GTGCAGGGTCCGAGGT-3' }\end{array}$ \\
\hline SMARCEI & $\begin{array}{l}\text { Forward } \\
\text { Reverse }\end{array}$ & $\begin{array}{l}\text { 5'-ATGGCCTTAGCTTAGGCT-3' } \\
\text { 5'-TTGGCAATGCCGTATTAGC-3' }\end{array}$ \\
\hline GAPDH & $\begin{array}{l}\text { Forward } \\
\text { Reverse }\end{array}$ & $\begin{array}{l}\text { 5'-AGCCACATCGCTCAGACAC-3' } \\
\text { 5'-GCCCAATACGACCAAATCC-3' }\end{array}$ \\
\hline U6 & $\begin{array}{l}\text { Forward } \\
\text { Reverse }\end{array}$ & $\begin{array}{l}\text { 5'-GCTTCGGCAGCACATATACTAAAAT-3' } \\
\text { 5'-CGCTTCACGAATTTGCGTGTCAT-3' }\end{array}$ \\
\hline
\end{tabular}

\section{Real-Time Quantitative PCR (RT-qPCR)}

Total RNA was extracted by Trizol (Invitrogen, USA). In each sample, $2 \mu \mathrm{g}$ RNA was used to synthesize cDNA as the templates of RT-qPCR using MMLV (Promega, Beijing, China). RT-qPCR was carried out in triplicate using a PrimeScript RT reagent kit (Takara, Japan) on the piko $^{\mathrm{TM}}$ Thermal Cycler (ThermoFisher, USA). Relative expression levels of genes were calculated using the $2^{-\Delta \Delta \mathrm{Ct}}$ method. U6 and GAPDH were used as internal controls for miRNA and mRNA, respectively. The sequences of primers are presented in

\section{Cell Transfection}

siRNAs against circVAPA siVAPA), 2 - $61 \quad 3 p$ overexpression plasmids (miP ,15-3p mics),, $2-615-3 p$ inhibitor and correspo ing ve cont is were purchased from Integrate Biotech angl, China). Lipo3000 (Invitrogen SA) as utilized or the subsequent transfection into cells.

\section{Ce Viabilit) Assay}

CCK (Sigma, SA) was utilized to measure cell viability. Eao of a 96-well plate was seeded with 2000 ce followed by an incubation of described time. ells were then added with CCK-8 solution and absorbance was measured at $450 \mathrm{~nm}$.

\section{Colony-Forming Assay}

Treated cells were seeded in 6-well plates with a density of 3000 cells per well, and cultured for 2 weeks. Then, methanol was utilized for fixing and $0.5 \%$ crystal violet 
was applied for staining. After $30 \mathrm{~min}$, the number of colonies were counted.

\section{Cell Apoptosis Assay}

Cell apoptosis was detected using Annexin V/Cell apoptosis staining kit (LMAI Bio, Shanghai, China), according to the protocol. The cell apoptosis was tested using FACScan flow cytometer (BD Biosciences, San Jose, CA).

\section{Transwell Assay}

Transwell chamber (Corning, USA) was utilized for migration and invasion assays. Cells were seeded in the upper inserts filled with RPMI-1640, and the lower chambers were filled with complete medium (10\% FBS). After 24 hours, cells suspended in the upper insets were removed. Cells in the lower chamber were fixed and stained with methanol and crystal violet. Migration cells were photographed using an inverted microscope (Olympus, Japan). For invasion assay, the upper chamber was precoated with Matrigel matrix (BD, USA).

\section{Western Blot}

Total protein was extracted from cells or tissues using lysis buffer (Thermo Fisher Scientific, USA). Equal amo of each sample were loaded on $10 \%$ SDS transferred on PVDF membranes. Afte olocked with 5, non-fat milk for an hour at room tamp ture nom were incubated at $4{ }^{\circ} \mathrm{C}$ overnigh ${ }^{+}$th spech primary antibodies against SMARCE1 00 , $1: 1000$-actin (Tiangen, 1:2000).
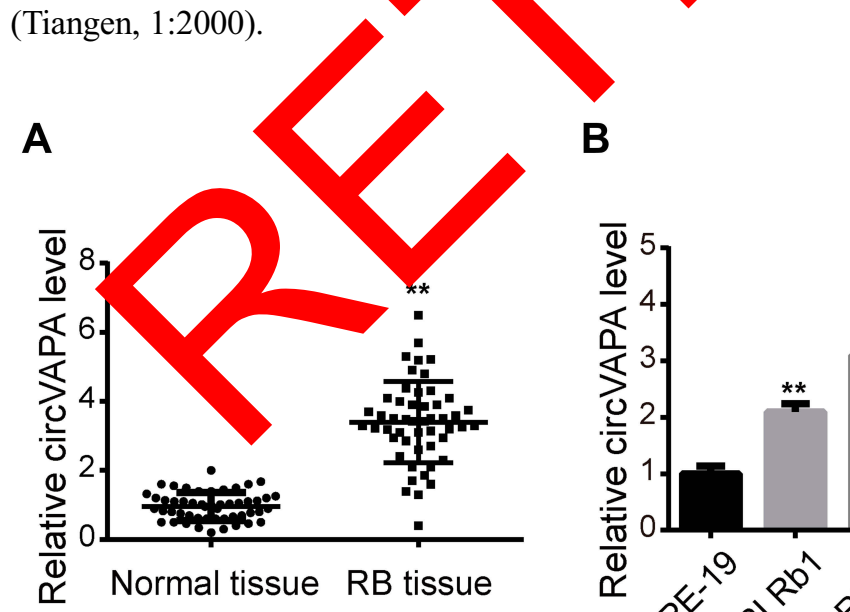

B

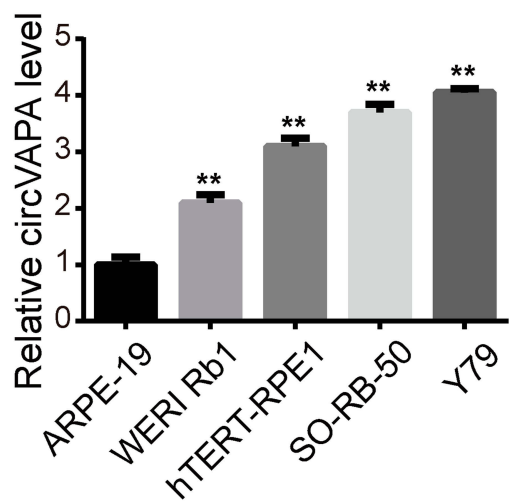

\section{Immunohistochemical Staining}

Tumors were treated with formalin and paraffin and sliced into $5 \mu \mathrm{M}$-thick sections. Xylene was utilized to deparaffinize the samples and ethanol was used for hydration. After blockage with serum for half an hour, samples were incubated with antibodies against SMARCE1 (1:200, Sigma) and Ki67 (1:500, Sigma) at $4{ }^{\circ} \mathrm{C}$ overnight. Secondary antibodies were taken for another incubation at room temperature for an hour. DBA was utilized for color reaction.

\section{Animal Model}

Mice (8-week-old) were pro ted by Zhej g Hospital of Integrated Traditional Ch' Wese a Wertern Iedicine. Y29 cells carrying sh-circl APA or sh-s $\mathrm{amb}^{\prime}$ (control group) were injected into $\mathrm{kks}$ of $y$ e mice. amor volumes were recorded week ${ }^{\prime}$ Fou tumors we removed in the absequent assays. Animal study s ap ved by Burcs Committee of Zhejiang Hosnital of Inte. ted Traditional Chinese and Western edicine and conduc.d in accordance with the Guidelines or Animal $C a$ in the National Institutes of Health.

Re

\section{A. YAPA Was Upregulated in RB and} Indicated Poor Prognosis

To identify expression patterns of circVAPA, qPCR was applied. As shown in Figure 1A and B, circVAPA expression levels were significantly increased both in RB patient tissue samples and cell lines. Correlations of $\operatorname{circVAPA}$ level with clinicopathological features were analyzed using Chi-squared

Figure I Expression level of circVAPA in RB. (A) circVAPA expressions in RB tissues and matched nearby normal ones were detected by qRT-PCR. (B) circVAPA expression levels in RB cell lines (WERI RbI, hTERT-RPEI, SO-RB-50 and Y79) and human retinal pigment epithelial cell line (ARPE-19) were examined by qRT-PCR. (C) Kaplan-Meier survival analysis was utilized to analyze the association of circVAPA expression and overall survival. $* * \mathrm{P}<0.01$. 
Table 2 Characteristics of RB Patients

\begin{tabular}{|l|l|l|l|l|}
\hline \multirow{2}{*}{$\begin{array}{l}\text { Characteristics } \\
\text { Total }\end{array}$} & $\mathbf{N}=\mathbf{5 0}$ & \multicolumn{2}{|l|}{ CircVAPA Expression } & P \\
\cline { 3 - 4 } & & $\begin{array}{l}\text { High } \\
(\mathbf{n}=\mathbf{3 3})\end{array}$ & $\begin{array}{l}\text { Low } \\
(\mathbf{n}=\mathbf{1 7})\end{array}$ & \\
\hline $\begin{array}{l}\text { Age (years) } \\
\geq 50 \\
<50\end{array}$ & 35 & 25 & 10 & 0.521 \\
\hline $\begin{array}{l}\text { Tumor size (cm) } \\
\geq 3\end{array}$ & 15 & 8 & 7 & \\
$<3$ & 28 & 18 & 10 & $0.032^{*}$ \\
\hline $\begin{array}{l}\text { FIGO stage } \\
\text { III-IV } \\
\text { I-II }\end{array}$ & 22 & 15 & 7 & \\
\hline $\begin{array}{l}\text { Lymph-node } \\
\text { metastasis } \\
\text { Yes } \\
\text { No }\end{array}$ & 18 & 13 & 5 & $0.025^{*}$ \\
\hline $\begin{array}{l}\text { Histological grade } \\
\text { Well } \\
\text { Moderately/poorly }\end{array}$ & 30 & 20 & 7 & \\
\hline
\end{tabular}

Note: $* \mathrm{P}<0.05$ means statistical difference.

test, and results (Table 2) indicated that circVAPA expression was correlated with tumor size $(\mathrm{P}=0.032)$, FIGO sta $(\mathrm{P}=0.025)$ and Lymph-node metastasis $(\mathrm{P}=0.036$ Moreover, higher circVAPA expression indicated poorer prognosis (Figure 1C). These findings suggested a po ntial oncogenic role of circVAPA.

\section{Knockdown of circVAP apresse} Proliferation and Metastasis \& RB Cells To investigate the effects circVAPA on $k$ we used sicircVAPA for loss-of-fy tion ex riments. Figure 2A shows that both the two siRNA nst circ $P A$ exerted knockdown efficienc. Fu tiona xpe nents were performed following si rcVAPA si-circVA,$A-2)$ transfection. Cell viability was mo ur bycoro assay, showing that the cell viabilities of $\mathrm{SO}, \mathrm{B}-50$ and Y79 cells were decreased after transfection of si-ch VAPA (Figure 2B). Colony-forming experiments also showed that the $\mathrm{RB}$ cell proliferation was suppressed with si-circVAPA transfection (Figure 2C). Whereas, the apoptotic rate of RB cells was increased upon circVAPA knockdown (Figure 2D). As metastasis was an evident feature of cancer cells, we further tested the effects of circVAPA on RB cell metastasis. As shown in Figure $2 \mathrm{E}$ and $\mathrm{F}$, in circVAPA knockdown groups, the migration and invasion abilities were restrained, compared with those in control groups. All these results suggested that $\operatorname{circVAPA}$ might play as a tumor promotional role in RB progression.

\section{circVAPA Served as a Sponge for miR- 6I5-3p}

We next explored the possible molecular mechanism. We utilized CircBank to predict $m i R-615-3 p$ as an interacting miRNA, with potential binding sites presented in Figure 3A. To confirm this prediction, we applied luciferase activity assay. As shown in Figure 3B, luciferase activity was suppressed with co-transfection of $m i P$ circVAPA, but not with co-transfe an of $m i \wedge \quad 15-3 p$ and mutant circVAPA. Moreover, mik $15-3 p$ expr sion was deceased when circVAPA s knocke down gure 3C). Further, we examined ession $m i R-3 p$ in $\mathrm{RB}$ cell lines, observing the dow agr ced lever of $m i R-615-3 p$ in SO-RB-50 and 9 cell lin in co parison with those in normal ARP 19 lls (Figure Also, miR-615-3p was downregulated in $\mathrm{Rb}$ issues, in comparison with those in norm cissues (Figure Additionally, the expression leve of circVAP4 and miR-615-3p in tumor tissues from 501 patients we detected by qPCR. Then, the correlation betwe circVA and miR-615-3p in RB tissues was deterined using rearson analysis. Results showed that miR-6153 vas satively correlated with $\operatorname{circVAPA}(3 \mathrm{~F})$.

\section{ircVAPA Exerted Oncogenic Effects on} RB Cells via Regulating miR-6/5-3p

We further investigated whether miR-615-3p participated in circVAPA regulation on RB. si-circVAPA was co-transfected with/without $m i R-615-3 p$ inhibitor, followed by functional experiments. We observed that $m i R-615-3 p$ inhibitor attenuated the reduction of cell viability and colony number induced by si-circVAPA (Figure 4A and B). The aggravation on cell apoptosis induced by si-circVAPA was also mitigated by $m i R-615-3 p$ inhibitor (Figure 4C). si-circVAPA downregulated the $\mathrm{RB}$ cell migration and invasion abilities, while $m i R-615-3 p$ inhibitor could partially reverse these alterations (Figure 4D and E). These observations indicated that $m i R-615-3 p$ was involved in the regulation.

\section{circVAPA Upregulated SMARCEI via miR-6I5-3p}

We utilized TargetScan network tool to predict SMARCE1 as the potential target of $m i R-615-3 p$ (Figure 5A). We applied luciferase activity assay to confirm this prediction. As shown in Figure 5B, luciferase activity was suppressed 
A

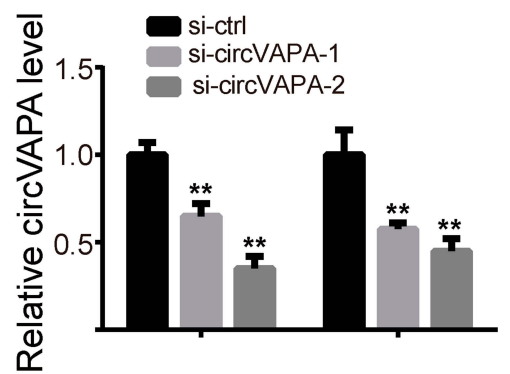

B

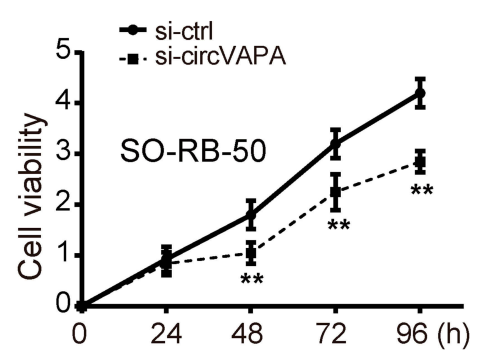

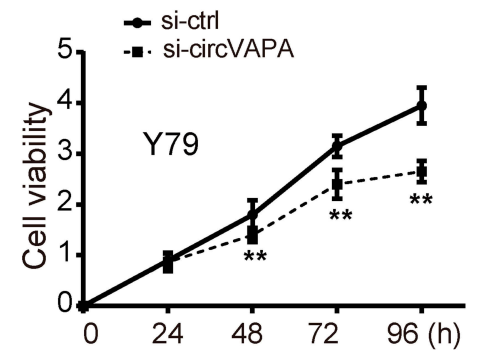

C

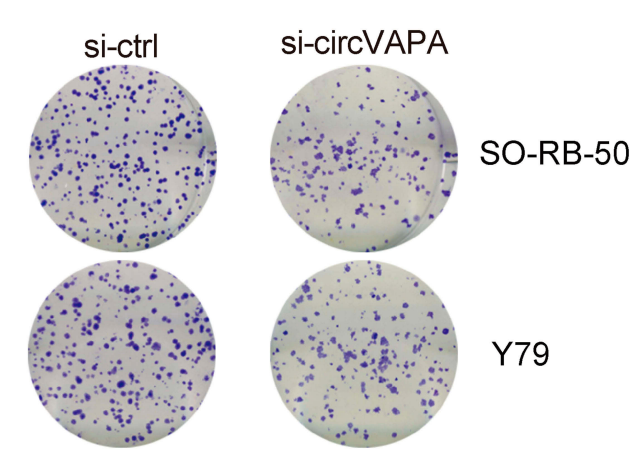

D
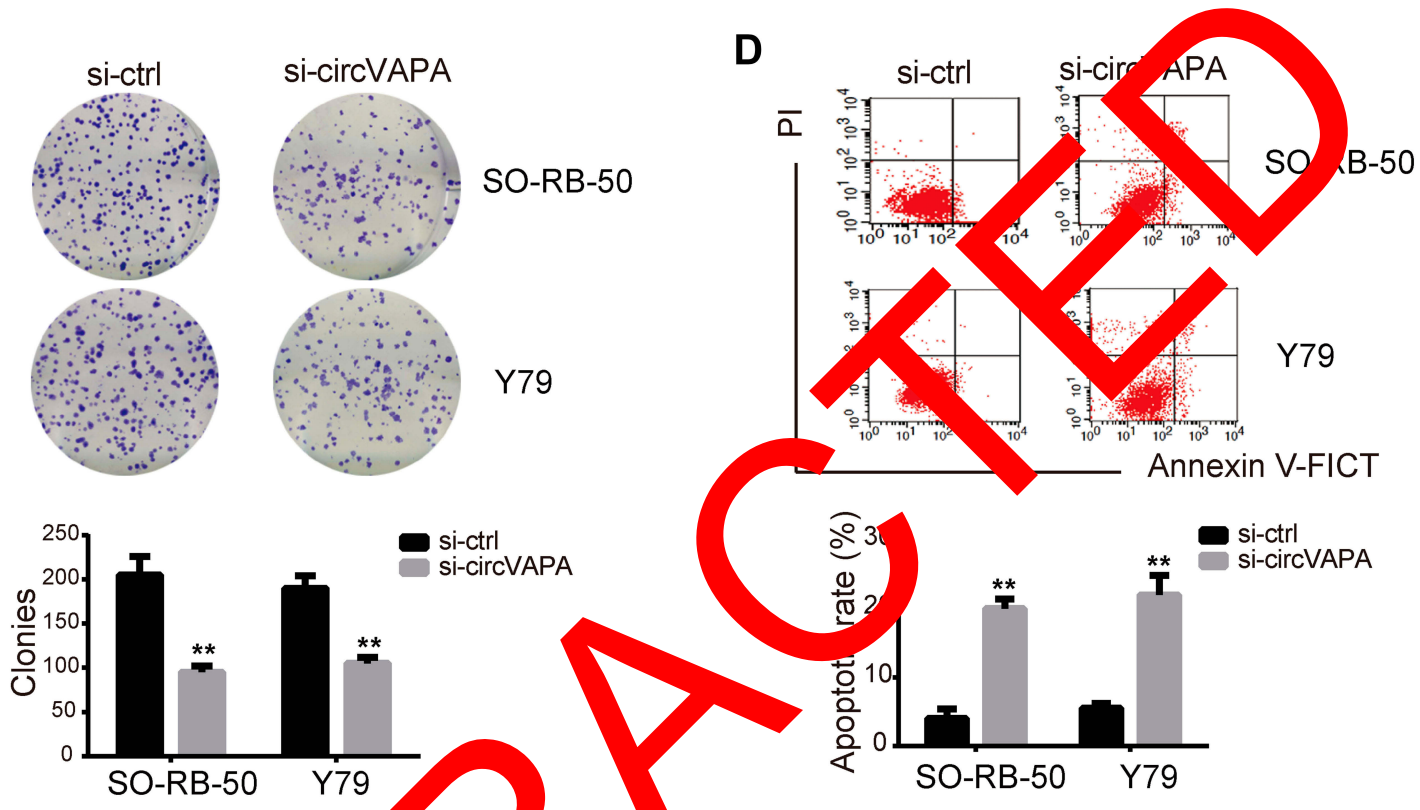

si-ctrl

si-circVAPA

E

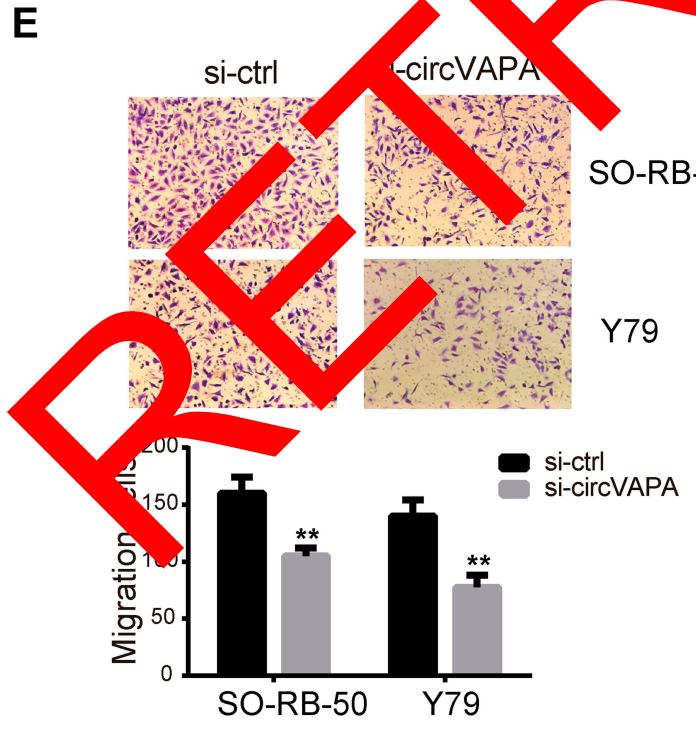

$\mathbf{F}$

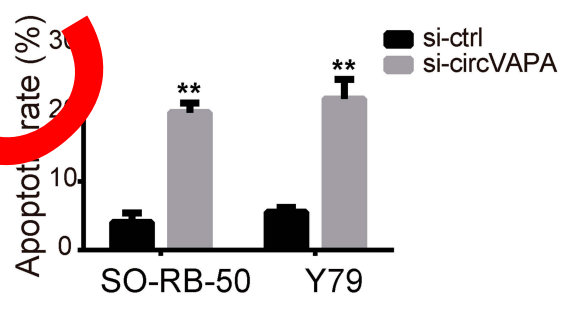

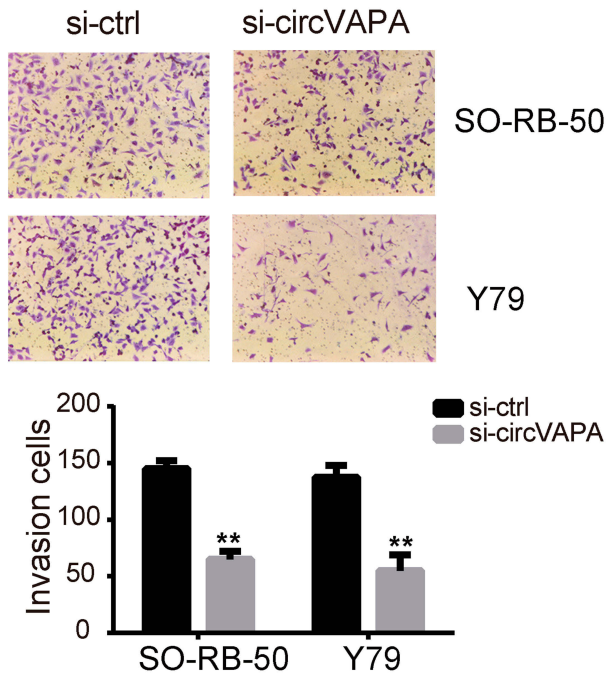

Figure 2 circVAPA exerted oncogenic effects on RB cells. (A) siRNAs against circVAPA were transfected into RB cell lines, and the knockdown efficiencies were tested by qRT-PCR. si-VAPA-2 was transfected into SO-RB-50 and Y79 cells. (B) CCK-8 assay was applied to assess cell viability. (C) Colony-forming experiments were performed to evaluate cell proliferation. (D) Flow cytometry was carried out to test cell apoptosis. (E) Transwell assays were utilized to detect cell migration. (F) Transwell assays were used to determine cell invasion. $* * \mathrm{P}<0.01$. 
A

$$
\begin{array}{cc}
\text { circVAPA (wt) } & \text { 5'-...CGGCAGGCGTTAGGGCTCGGG...-3' } \\
\text { miR-615-3p I I I | I I I } & \text { 3'-UUCUCCCUCUGGGUCCGAGCCU -5' }
\end{array}
$$

circVAPA (mut) 5'-...CGGCAGGCGTTAGUUUAACCG...-3'

B
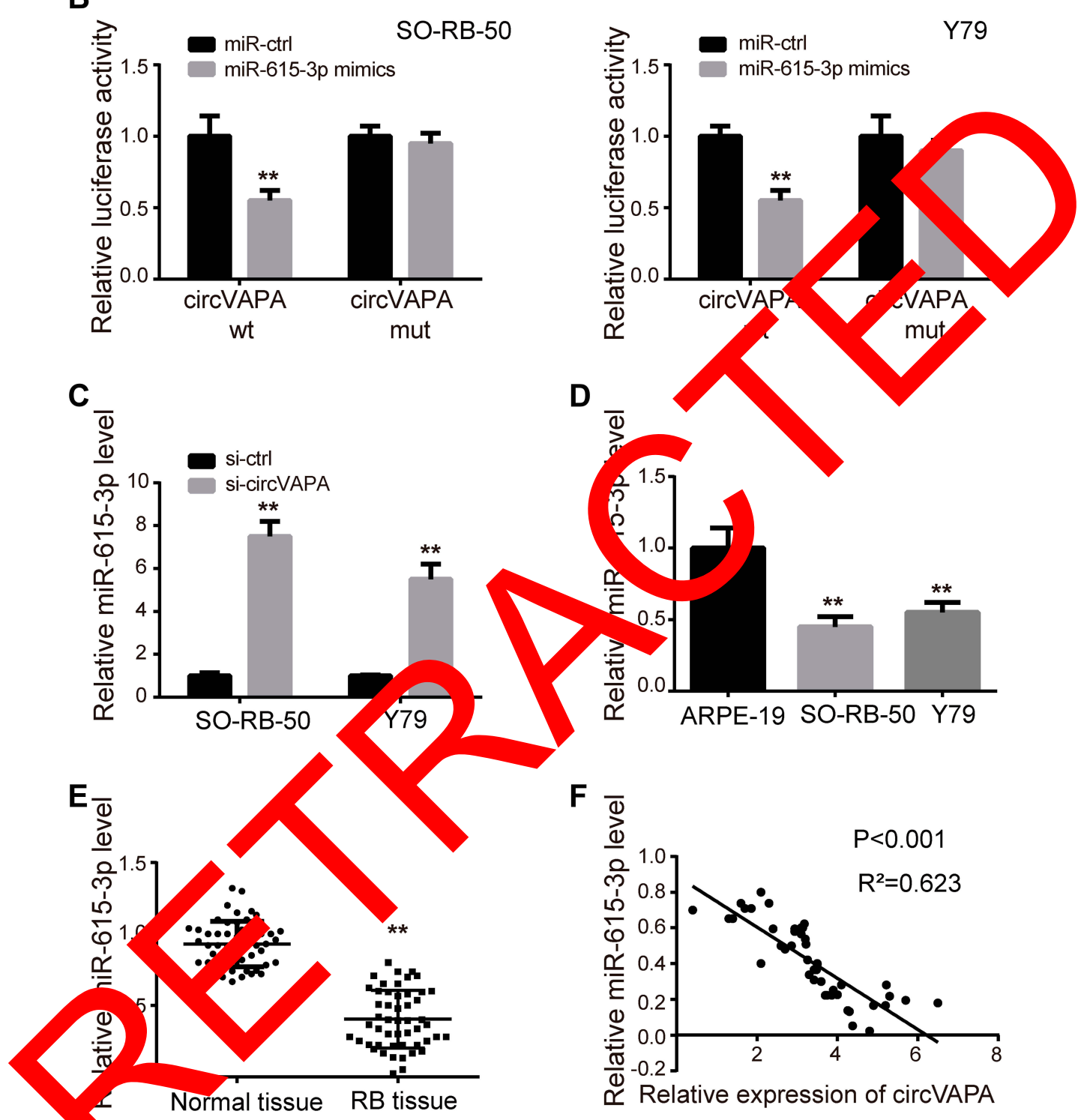

D
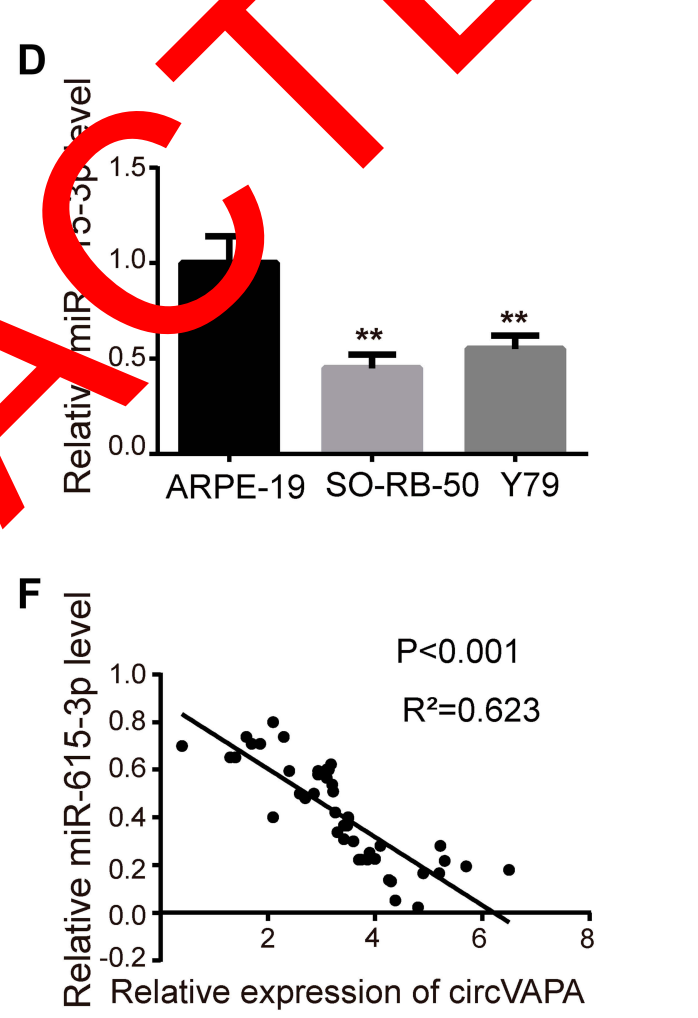

Figure 3 circVAPA was a Sp for miR-6/5-3p. (A) Putative interacting sites predicted by CircBank. (B) Luciferase activities were detected after co-transfection of miR-6/5$3 p$ and circVAPA wt/mut. (C) si-circVAPA was transfected, and miR-6/5-3p expression was measured by qRT-PCR. (D) miR-6/5-3p expression levels in RB cell lines (SO-RB-50 and Y79) and normal cell line (ARPE-19) were measured by RT-qPCR. (E) miR-6/5-3p expression levels in RB tissues and matched nearby normal ones were measured by RTqPCR. (F) Pearson's correlation analysis of circVAPA and miR-6I5-3p in RB tissue. $* * P<0.01$.

with co-transfection of $m i R-615-3 p$ and wild type 3'UTR of SMARCE1, but not with co-transfection of $m i R-615-3 p$ and mutant $3^{\prime} \mathrm{UTR}$ of SMARCE1. Moreover, the expression level of SMARCE1 was downregulated by $m i R-615$ $3 p$ overexpression (Figure $5 \mathrm{C}$ ). These results confirmed that $m i R-615-3 p$ directly interacted with SMARCE1. In addition, we observed that SMARCE1 was upregulated in $\mathrm{RB}$ tissues, in comparison with those in normal tissues (Figure 5D). Further, the mRNA and protein expression levels of SMRCE1 were downregulated by si-circVAPA, 
A

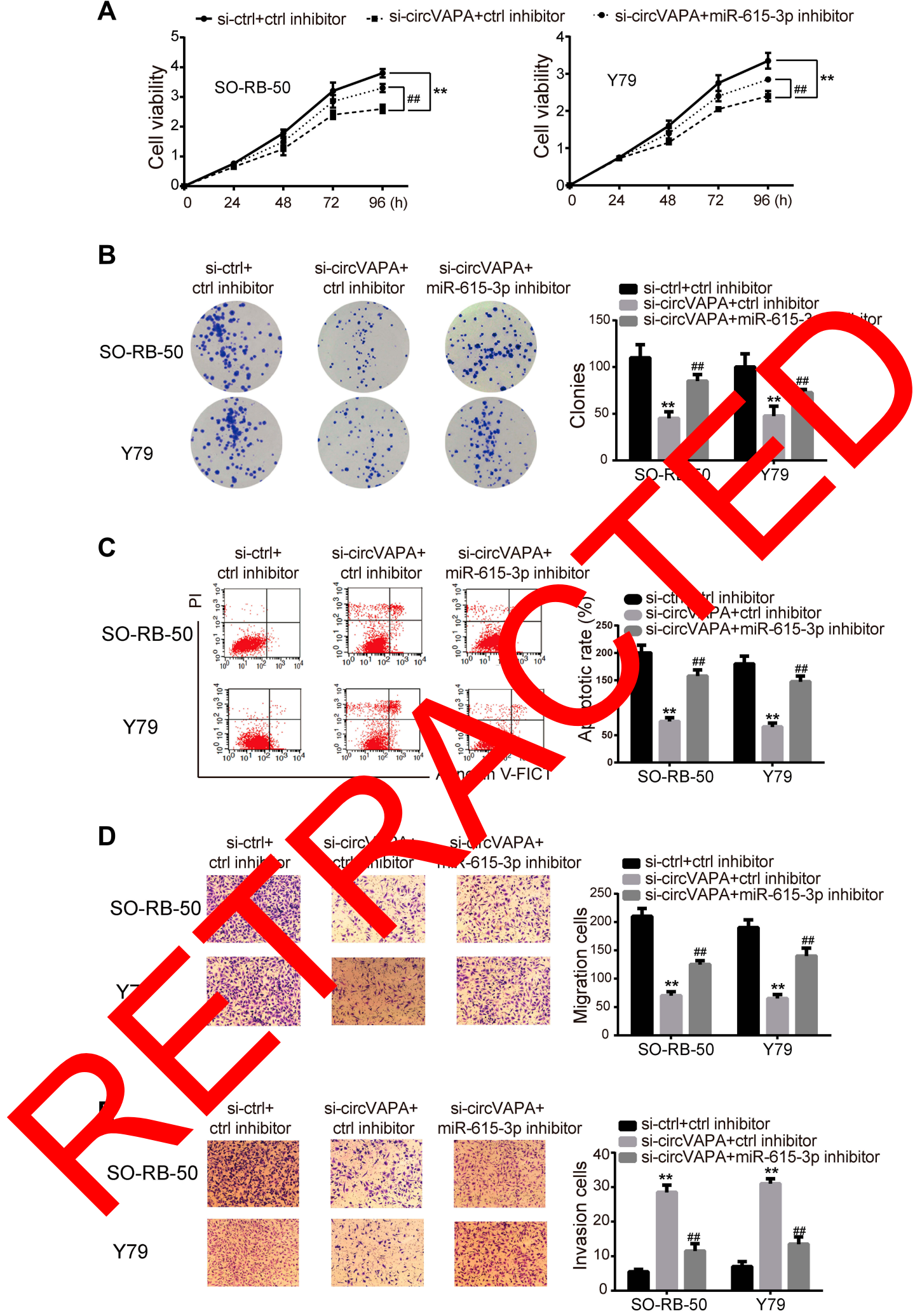

- si-circVAPA+miR-615-3p inhibitor

Figure 4 miR-6/5-3p inhibitor mitigated the si-circVAPA induced oncogenic effects on RB cells. si-circVAPA was co-transfected with miR-6/5-3p inhibitor or its negative control inhibitor. (A) CCK-8 assay was applied to assess cell viability. (B) Colony-forming experiments were performed to evaluate cell proliferation. (C) Flow cytometry was carried out to test cell apoptosis. (D) Transwell assays were utilized to detect cell migration. (E) Transwell assays were used to determine cell invasion. $* *$ P $<0.0 \mathrm{I}$ compared with si-ctrl+ctrl inhibitor; \#\#P $<0.01$ compared with si-circVAPA+ctrl inhibitor. 
A

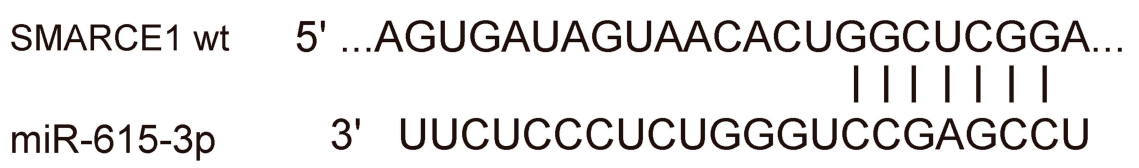

SMARCE1 mut $\quad 5^{\prime}$...AGUGAUAGUAACACUCCAAACCA...

B
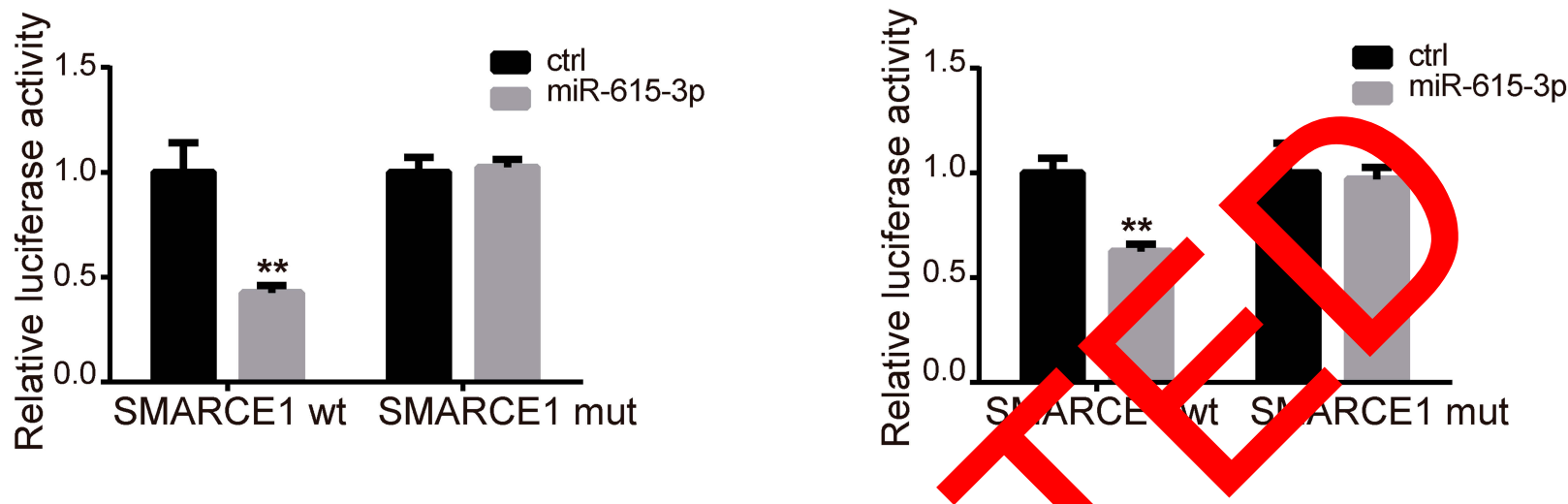

C

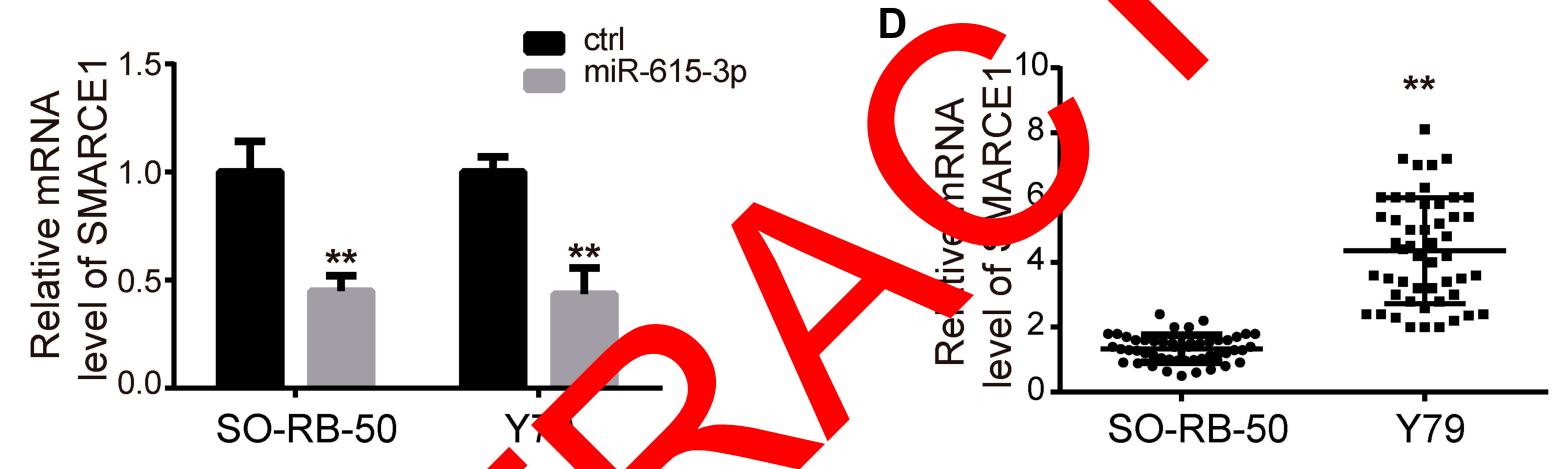

$\mathbf{E}$

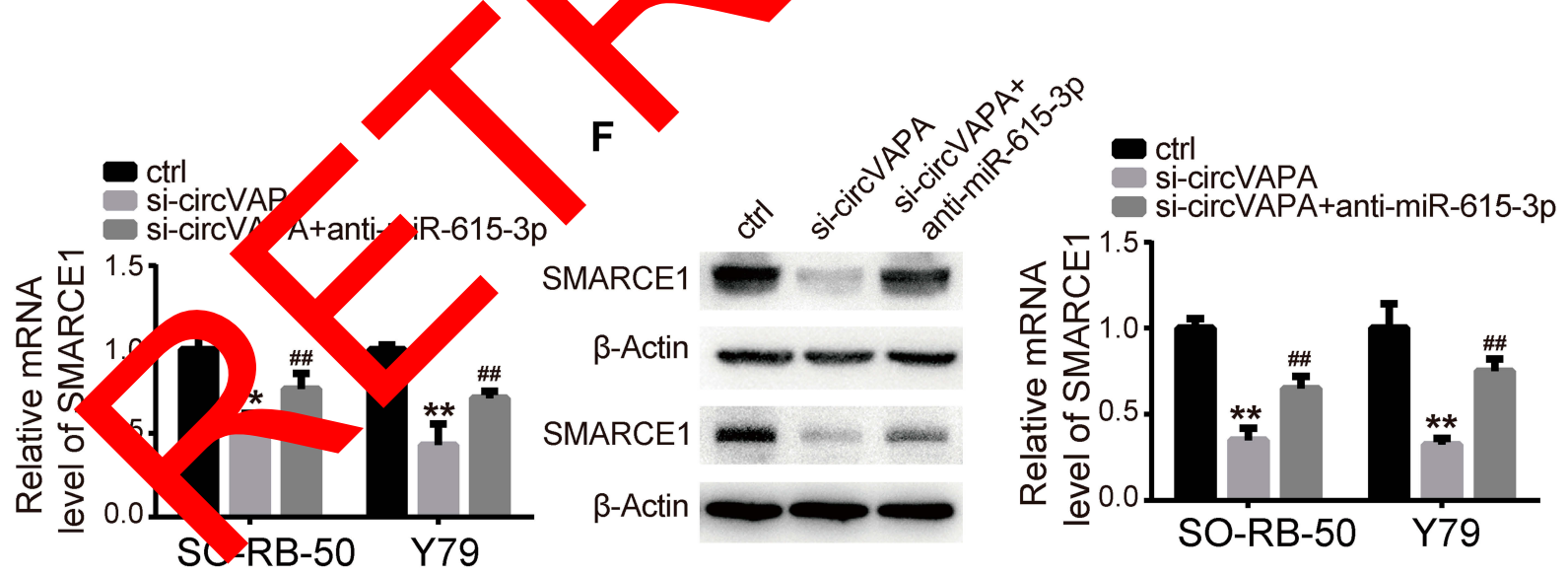

Figure 5 SMARCEI was positively modulated by circVAPA via miR-615-3p. (A) Putative interacting sites predicted by TargetScan. (B) Luciferase activities were detected after co-transfection of miR-6/5-3p and SMARCEI 3'UTR wt/mut. (C) miR-6/5-3p was overexpressed, and miR-6/5-3p level was measured by qRT-PCR. (D) SMARCEI expression levels in RB tissues and matched nearby normal ones were measured by RT-qPCR. (E, F) si-circVAPA was co-transfected with miR-6/5-3p inhibitor or negative control inhibitor, then SMARCEI mRNA and protein levels were determined by qRT-PCR and Western blot. **P<0.0I compared with control group; \#P<0.0I compared with sicircVAPA group. 
while $m i R-615-3 p$ inhibitor could partially reverse these alterations (Figure $5 \mathrm{E}$ and $\mathrm{F}$ ). All the above observations indicated that $\operatorname{circ} V A P A$ sponged $m i R-615-3 p$ to positively regulate $S M A R C E 1$ gene expression, which might be the underlying mechanism.

\section{circVAPA Silencing Hampered RB Tumorigenesis in vivo}

Tumor xenografts were generated in mice to perform in vivo experiments. Y79 cells expressing sh-circVAPA or sh-scramble (ctrl) were injected into mice. Tumor size and tumor weight were detected weekly and 4 weeks later, respectively (Figure 6A and B). After tumors were removed 4 weeks later, we examined the gene expressions in tumor tissues. RTqPCR results showed that circVAPA and SMARCE1 were downregulated in $\operatorname{circVAPA}$-depleted mice, while miR-615-3p was upregulated (Figure 6C-E). Immunohistochemistry assays were further performed to determine $S M A R C E 1$ and Ki67 expression levels. SMARCE1 was downregulated in shcircVAPA mice, in line with the qPCR results we found (Figure 6F). Ki67, a cell proliferation marker, was also lowly expressed in sh-circVAPA mice (Figure 6G). The in vivo observations were consistent with those found in cell lines.

\section{Discussion}

$\mathrm{RB}$ is of high mortality rate that was commonly happened in children under five years old. ${ }^{13}$ Despite the great efforts in treating this disease, the survival row, especially in less developed countries. ${ }^{14} \mathrm{He}$, it is urge to find out new approaches to overcome RB. E r ring evide e showed that circRNAs were dysre ated and yyed tumor promotional or anti-cance oles ir zancer gressions. ${ }^{15}$ As to $\mathrm{RB}$, there exister se al ports demonstrating the relationship between arcRNAs d RB -velopment. For example,

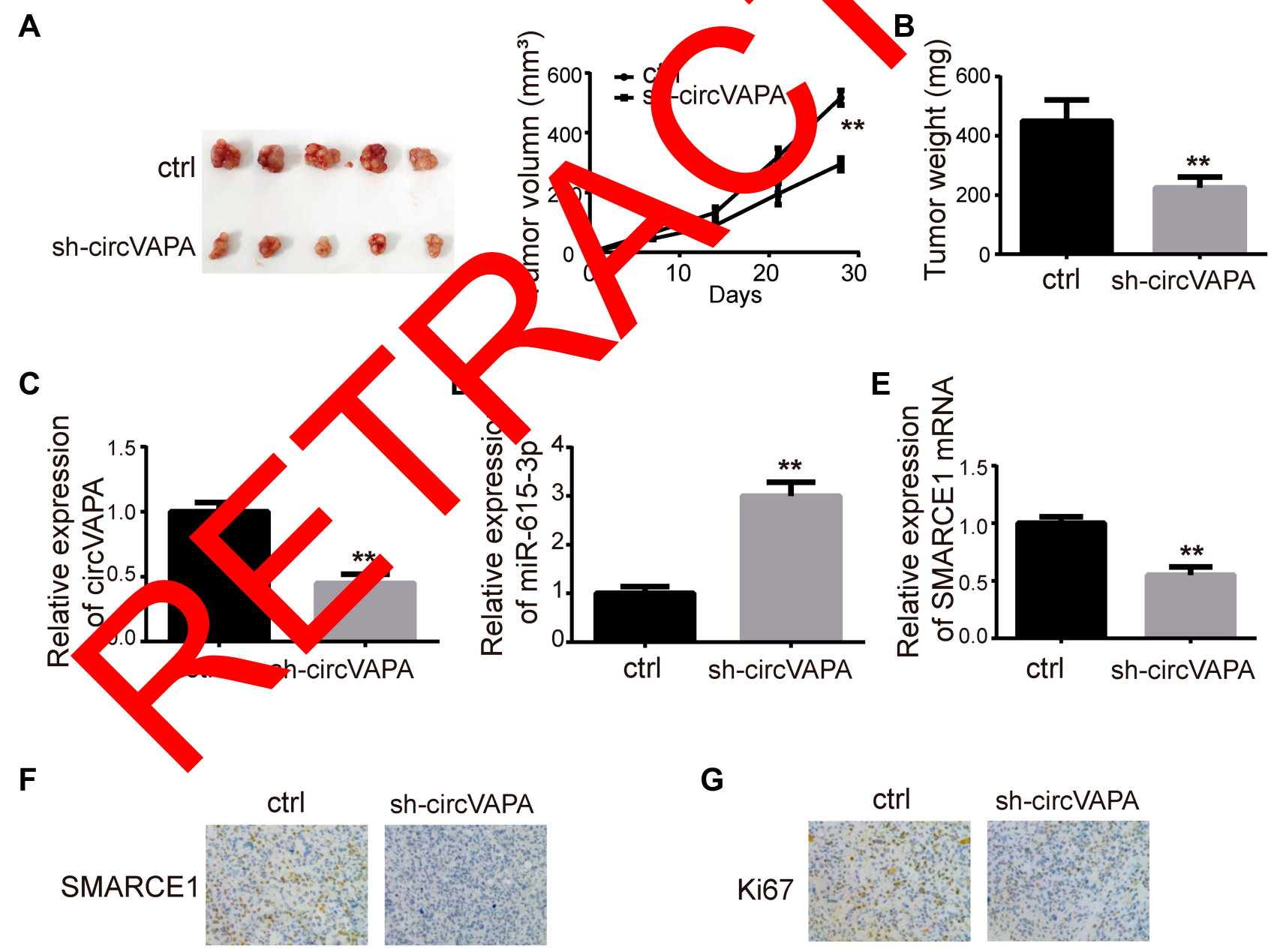

Figure 6 circVAPA silencing hampered RB tumorigenesis in mice. Mice were injected with Y79 cells expressing sh-circVAPA or sh-scramble stably. (A, B) Image of tumors removed 4 weeks later and the corresponding tumor sizes and weights. (C) circVAPA expression level in sh-circVAPA and sh-scramble mice. (D) miR-6 I 5-3p expression levels in sh-circVAPA and sh-scramble mice. (E) SMARCEI mRNA levels in sh-circVAPA and sh-scramble mice. (F) SMARCEI expression levels were detected by ICH. (G) Ki67 expression levels were assessed by ICH. $* * \mathrm{P}<0.01$. 
circ0001694 was revealed to overexpressed in human RB tissue and indicated poor survival rate by modulating AKT/ mTOR signaling pathway. ${ }^{16}$ Circ0006168 was reported to activate S6K/S6 signaling and modulate miR-384/RBBP7, thereby contributing to $\mathrm{RB}$ cell viability and metastasis. ${ }^{17}$ Thus, in this study, we aimed to investigate the influence of circRNA in RB pathogenesis.

In this study, we applied qRT-PCR to determine the expression profile of circVAPA in RB. Results showed that circVAPA was highly expressed in RB tissue and cell lines. This result prompted us to explore the function of $\operatorname{circ} V A P A$ in $\mathrm{RB}$ further. In vitro, knockdown of circVAPA inhibited $\mathrm{RB}$ cell viability and colony formation ability, as well as enhanced cell apoptosis. Moreover, the migration and invasion of SO-RB-50 and Y79 cells were significantly decreased after si-circVAPA were transfected. Of note, we detected the cell apoptosis, migration and invasion $24 \mathrm{~h}$ after transfection, when the cell viability had not been affected (the cell viability curve shown in Figure 2B). Thus, the reduced cell viability would not influence the phenotypic changes. These results suggested that circVAPA exerted a significant influence on RB cell proliferation, migration and invasion.

Further, we aimed to explore the possible mechanisms. More and more reports confirmed that circRNAs were rich miRNA binding sites and likely to function by acting a sponges of miRNAs, thereby releasing the downstrm target genes of miRNAs. ${ }^{18,19}$ For example, $B C R C$-3 ribitec ladder cancer through sequestering $m i R-182-$ and $\mathrm{p} c$ sirolv regulating $p 27 .{ }^{20}$ Circ103869 downregy 'ed $m$. 2-3p and released FOXO4 mRNA, thereby ributing to lorectal cancer cell proliferation. ${ }^{21}$ Circ06 4043 regulated Shail via sponging $m i R-153-3 p$ to pro ste malignan elanoma. ${ }^{22}$ In this study, we predicted binding sites of creVAPA and $m i R-615-3 p$ by inform ics s cware and confirmed that circVAPA could $b-1$ to $m$ 615-3p luciferase reporter activity. Mor er, $\mathrm{n}$ detec expression pattern of $m i R-615-3$ RB ce thand and tissues, and found that 615-3p was low cownregulated in RB cell lines and tissues, which was in line the previous prediction. Also, in RB tissues, there existed ar regative correlation between circVAPA and $m i R-615-3 p$ analyzed by Pearson correlation analysis. Further, we conducted functional experiments to confirm that miR-615-3p was involved in the regulation mechanisms. si-circVAPA was co-transfected with $m i R-615-3 p$ inhibitor or ctrl inhibitor. Then, CCK-8, colony formation assay, apoptosis assay and transwell assay were conducted. We found that sicircVAPA suppressed RB cell proliferation, migration and invasion, as well as promoted RB cell apoptosis, while $m i R$ - 615-3p inhibitor could partially reverse these changes. These results suggested that $\operatorname{circ} V A P A$ regulated $\mathrm{RB}$ cell proliferation and metastasis by sponging $m i R-615-3 p$.

MicroRNAs are known to bind to target genes' 3'UTR, thus downregulate their expressions. ${ }^{23}$ Many miRNAs participate in cancer progression through repressing target mRNA expressions. For example, miR-376a-3p targeted KLF15 to promote colorectal cell proliferation and metastasis. ${ }^{24} \mathrm{miR}$ 498 promoted RB cell proliferation and inhibited cell apoptosis via targeting CCPG1. ${ }^{25}$ Notch 1 and $P A X 6$ were suppressed to express normally by $m i R-432$ reby hampering RB progression. ${ }^{26}$ Herein, we show SMARQ as a direct target of $m i R-615-3 p$ and found th SMARCE 1 as highly expressed in RB tissue. $S M A E 1$ wa tumor omoter in many types of cancers ach as pvarla cer, ${ }^{27}$ breast cancer, $^{28}$ gastric cance 9 an ${ }^{\prime}$ Mepatoma carcinoma. ${ }^{30}$ In the present work ve pre ed $S M A C E 1$ as a target of $m i R-615-3 p$ b vetScan sol followed by luciferase reporter activity assa detecte expressio of SMARCE1, and found that SM RCE1 was upregulated in RB tissues, which might be inh ited by $m i R-15-3 p$. Furthermore, we wanted to investigat vether $c i$ VAPA could regulate $S M A R C E 1$ via $m i R$ 615-3p. $\triangle A P A$ was co-transfected with miR-615-3p in $\quad$ or ctrl inhibitor, and SMARCE1 expression was etected. Results showed that SMARCE1 was suppressed by si-circVAPA, while the suppression was partially reversed miR-615-3p inhibitor. These findings indicated that miR615-3p/SMARCE1 axis might be involved in circVAPA regulated $\mathrm{RB}$ progression. To conform this supposition, we carried out in vivo experiments. Tumors in circVAPA knockdown group were smaller and lighter, in comparison with those in the control group. Importantly, miR-615-3p expression level was upregulated in $\operatorname{circ} V A P A$ knockdown mice, while $S M A R C E 1$ expression level was downregulated. All the results suggested that $\operatorname{circ} V A P A$ promoted RB progression via regulating miR-615-3p/SMARCE1.

\section{Conclusion}

CircVAPA promoted RB cell proliferation, migration and invasion, as well as inhibited cell apoptosis. In terms of mechanism, circVAPA could positively regulate SMACE1 via sponging $m i R-615-3 p$. This finding might provide new targets for clinical diagnosis and therapy of RB.

\section{Disclosure}

The author declares that they have no conflict of interest in this work. 


\section{References}

1. Dimaras H, Kimani K, Dimba EA, et al. Retinoblastoma. Lancet. 2012;379(9824):1436-1446. doi:10.1016/S0140-6736(11)61137-9

2. Singh G, Daniels AB. Disparities in retinoblastoma presentation, treatment, and outcomes in developed and less-developed countries. Semin Ophthalmol. 2016;31(4):310-316. doi:10.3109/08820538.2016.1154177

3. Meng S, Zhou H, Feng Z, et al. CircRNA: functions and properties of a novel potential biomarker for cancer. Mol Cancer. 2017;16(1):94. doi:10.1186/s12943-017-0663-2

4. Wu J, Qi X, Liu L, et al. Emerging epigenetic regulation of circular RNAs in human cancer. Mol Ther Nucleic Acids. 2019;16:589-596. doi:10.1016/j.omtn.2019.04.011

5. Memczak S, Jens M, Elefsinioti A, et al. Circular RNAs are a large class of animal RNAs with regulatory potency. Nature. 2013;495 (7441):333-338. doi:10.1038/nature11928

6. Chen -L-L, Yang L. Regulation of circRNA biogenesis. RNA Biol. 2015;12(4):381-388. doi:10.1080/15476286.2015.1020271

7. Szabo L, Salzman J. Detecting circular RNAs: bioinformatic and experimental challenges. Nat Rev Genet. 2016;17(11):679-692. doi:10.1038/nrg.2016.114

8. Glazar P, Papavasileiou P, Rajewsky N. circBase: a database for circular RNAs. RNA. 2014;20(11):1666-1670. doi:10.1261/rna.043687.113

9. Hou L-D, Zhang J. Circular RNAs: an emerging type of RNA in cancer. Int J Immunopathol Pharmacol. 2017;30(1):1-6. doi:10.1177/ 0394632016686985

10. Li X-N, Wang Z-J, Ye C-X, et al. Circular RNA circVAPA is upregulated and exerts oncogenic properties by sponging miR-101 in colorectal cancer. Biomed Pharmacother. 2019;112:108611. doi:10.1016/j.biopha.2019.108611

11. Liu C, Zhong X, Li J, et al. Circular RNA circVAPA promotes cell proliferation in hepatocellular carcinoma. Hum Gene Ther 2019;30(4):152-159. doi:10.1089/humc.2019.079

12. Zhou S-Y, Chen W, Yang S-J, et al. Circular RNA circVAP. ulates breast cancer cell migration and invasion 130a-5p. Epigenomics. 2020;12(4):303-317. A0.22 /epi-20 0124

13. Benavente CA, Dyer MA. Genetics and epr etics blastoma. Annu Rev Pathol. 2015 (1):5 doi:10.1146/ annurev-pathol-012414-040259

14. Ortiz MV, Dunkel IJ. Reting o. J Child ^ 2016;31 (2):227-236. doi:10.1177/088 /3815, 43

15. Vo JN, Cieslik M, Zhang et al. The lan ape of circular RNA in cancer. Cell. $20,176(4): 869-881 \quad 3 . \quad$ doi:10.1016/j. cell.2018.12.021

16. Xing L, Zhang L, ng Y al. Downregulation of circular RNA hsa_circ_0001649 ino poor pro osis for retinoblastoma and regulates feratio nd ap osis via AKT/mTOR signaling pathwa Biomed harmac 2018;105:326-333. doi:10.1016/j. iop 2018.05,

OncoTargets and Therapy

\section{Publish your work in this journal}

OncoTargets and Therapy is an international, peer-reviewed, open access journal focusing on the pathological basis of all cancers, potential targets for therapy and treatment protocols employed to improve the management of cancer patients. The journal also focuses on the impact of management programs and new therapeutic

Submit your manuscript here: https://www.dovepress.com/oncotargets-and-therapy-journal
17. Xie Z-F, Li H-T, Xie S-H, et al. Circular RNA hsa_circ_0006168 contributes to cell proliferation, migration and invasion in esophageal cancer by regulating miR-384/RBBP7 axis via activation of S6K/S6 pathway. Eur Rev Med Pharmacol Sci. 2020;24(1):151-163. doi:10.26355/eurrev_202001_19906

18. Kulcheski FR, Christoff AP, Margis R. Circular RNAs are miRNA sponges and can be used as a new class of biomarker. J Biotechnol. 2016;238:42-51. doi:10.1016/j.jbiotec.2016.09.011

19. Zhang X, Zhu M, Yang R, et al. Identification and comparison of novel circular RNAs with associated co-expression and competing endogenous RNA networks in pulmonary tuberculosis. Oncotarget. 2017;8(69):113571-113582. doi:10.18632/oncotarget.22710

20. Xie F, Li Y, Wang M, et al. Circular RNA BCRC-3 suppresses bladder cancer proliferation through miR-182-5p/p27 axis. Mol Cancer. 2018;17(1):144. doi:10.1186/-102018-0892-z

21. Bian L, Zhi X, Ma L, et al. Hsa_ci CNA_to 99 regulated the cell proliferation and migration in orectal cance ia miR-532-3p / FOXO4 axis. Biochem Biophys Commun. 20 505(2):346-352. doi:10.1016/j.bbrc.2018.

22. Luan W, Shi Y, Zhou 7 al. circRN 0840 promote malignant melanoma progress via miR $3-3 \mathrm{p} / \mathrm{S}$ xis. Biochem Biophys Res Commun. 2010 2(1):? 29. doi:10.1016/j.bbrc.2018.05.114

23. Mohr AM, N JL. Lew of mi RNA biology. Semin Liver Dis. 2015\% (1):3-11. do, 1055,034-1397344

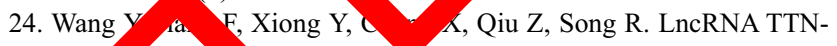
AS1 s,onges h $376 \mathrm{a}-3 \mathrm{p}$ to promote colorectal cancer progression via upregulating \ 15. Life Sci. 2019;244:116936.

25 callg, Wei N, Wan, et al. miR-498 promotes cell proliferation and inhibits cell apoptosis in retinoblastoma by directly targeting CCPG1. I ds Nerv Syst'. 2018;34(3):417-422. doi:10.1007/ s00381-017, 22-8

i X, Yar L, Shuai T, et al. MiR-433 inhibits retinoblastoma by suppressing Notch1 and PAX6 expression. Biomed Pharmacother. 2016;82:247-255. doi:10.1016/j.biopha.2016.05.003

27. nnakakis A, Karapetsas A, Dangaj D, et al. Overexpression of SMARCE1 is associated with CD8+ T-cell infiltration in early stage ovarian cancer. Int J Biochem Cell Biol. 2014;53:389-398. doi:10.1016/j.biocel.2014.05.031

28. Sethuraman A, Brown M, Seagroves TN, et al. SMARCE1 regulates metastatic potential of breast cancer cells through the HIF1A/PTK2 pathway. Breast Cancer Res. 2016;18(1):81. doi:10.1186/s13058016-0738-9

29. Liu H, Zhao Y-R, Chen B, et al. High expression of SMARCE1 predicts poor prognosis and promotes cell growth and metastasis in gastric cancer. Cancer Manag Res. 2019;11:3493-3509. doi:10.2147/ CMAR.S195137

30. Wu H-J, Zhuo Y, Zhou Y-C, et al. miR-29a promotes hepatitis B virus replication and expression by targeting SMARCE1 in hepatoma carcinoma. World J Gastroenterol. 2017;23(25):4569-4578. doi:10.3748/wjg.v23.i25.4569 agents and protocols on patient perspectives such as quality of life, adherence and satisfaction. The manuscript management system is completely online and includes a very quick and fair peer-review system, which is all easy to use. Visit http://www.dovepress.com/ testimonials.php to read real quotes from published authors. 